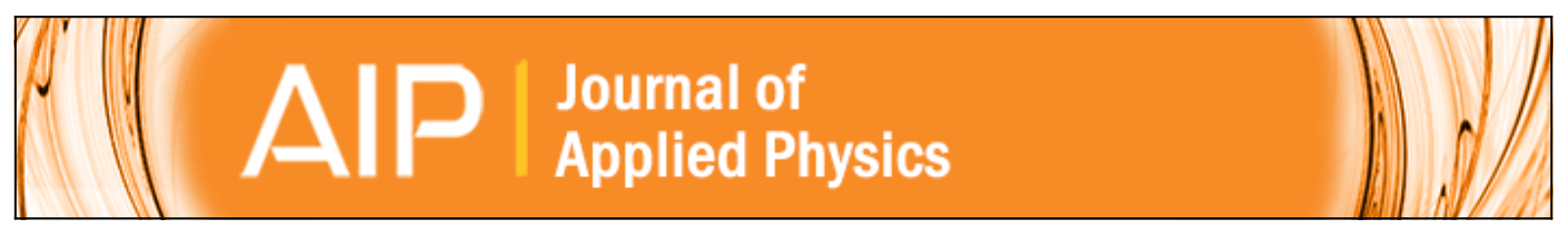

\title{
Enhancing the sensitivity of magnetoelectric sensors by increasing the operating frequency
}

Jonathan Petrie, Dwight Viehland, David Gray, Sanjay Mandal, Gollapudi Sreenivasulu, Gopalan Srinivasan, and Alan S. Edelstein

Citation: Journal of Applied Physics 110, 124506 (2011); doi: 10.1063/1.3668752

View online: http://dx.doi.org/10.1063/1.3668752

View Table of Contents: http://scitation.aip.org/content/aip/journal/jap/110/12?ver=pdfcov

Published by the AIP Publishing

\section{Articles you may be interested in}

Enhanced sensitivity in magnetoelectric current-sensing devices with frequency up-conversion mechanism by modulating the magnetostrictive strain

J. Appl. Phys. 115, 17E505 (2014); 10.1063/1.4862081

Enhanced sensitivity of magnetoelectric sensors by tuning the resonant frequency

Appl. Phys. Lett. 99, 043504 (2011); 10.1063/1.3617428

Low damping resonant magnetoelectric sensors

Appl. Phys. Lett. 97, 152503 (2010); 10.1063/1.3497277

dc magnetoelectric sensor based on direct coupling of Lorentz force effect in aluminum strip with transverse piezoelectric effect in $0.7 \mathrm{~Pb}(\mathrm{Mg} 1 / 3 \mathrm{Nb} 2 / 3) \mathrm{O} 3-0.3 \mathrm{PbTiO} 3$ single-crystal plate

J. Appl. Phys. 107, 09E702 (2010); 10.1063/1.3337748

Modeling and the signal-to-noise ratio research of magnetoelectric sensors at low frequency

Appl. Phys. Lett. 91, 142905 (2007); 10.1063/1.2794435

MIT LINCOLN

LABORATORY CAREERS

Discover the satisfaction of innovation and service

to the nation
- Space Control

- Air \& Missile Defense

- Communications Systems \& Cyber Security

- Intelligence, Surveillance and

Reconnaissance Systems

- Advanced
Electronics
- Tactical Systems
- Homeland
Protection
- Air Traffic Control

LINCOLN LABORATORY

MassachusetTs Institute of TeChNOLOGY

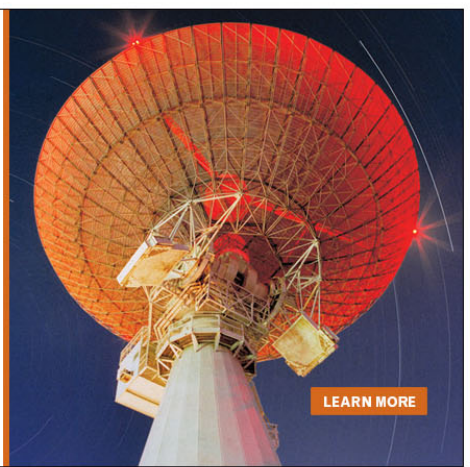




\title{
Enhancing the sensitivity of magnetoelectric sensors by increasing the operating frequency
}

\author{
Jonathan Petrie, ${ }^{1}$ Dwight Viehland, ${ }^{2}$ David Gray, ${ }^{2}$ Sanjay Mandal, ${ }^{3}$ Gollapudi Sreenivasulu, ${ }^{3}$ \\ Gopalan Srinivasan, ${ }^{3}$ and Alan S. Edelstein ${ }^{1, a)}$ \\ ${ }^{1}$ US Army Research Laboratory, Adelphi, Maryland 20783, USA \\ ${ }^{2}$ Virginia Tech University, Blacksburg, Virginia 24060, USA \\ ${ }^{3}$ Oakland University, Rochester, Michigan 48309, USA
}

(Received 2 June 2011; accepted 16 October 2011; published online 20 December 2011)

We present a field modulation technique that increases the operating frequency of magnetoelectric (ME) sensors so that it can match the mechanical resonance frequency of the sensor. This not only improves the sensitivity but also reduces the effect of $1 / f$ noise that is inherent at low frequencies. The technique, which is shown to apply to both symmetric and asymmetric ME sensors, relies on the strong, nonlinear magnetic field dependence of the magnetostriction. The combination of a lower $1 / f$ noise and enhanced response at resonance has increased the signal to noise ratio of a symmetric sensor by two orders of magnitude. The detection limit of this sensor was lowered from 90 to $7 \mathrm{pT} / \sqrt{\mathrm{Hz}}$ at $1 \mathrm{~Hz}$ in a magnetically unshielded environment. (C) 2011 American Institute of Physics. [doi:10.1063/1.3668752]

\section{INTRODUCTION}

Magnetoelectric (ME) sensors ${ }^{1}$ are passive sensors with sensitivities that are generally better than all but the most expensive, current magnetic sensors. ME sensors are composed of layers of mechanically coupled magnetoelectric and magnetostrictive material. In a magnetic field, the magnetostrictive material exerts a stress on the piezoelectric material, which in turn generates a charge. The geometry of the layers in a ME sensor may be symmetric (e.g., Metglas-lead zirconate titanate (PZT)-Metglas trilayer) or asymmetric (e.g., Metglas-PZT bilayer) about a central plane parallel to the layers. Because their output depends on the amount of strain, larger ME sensors with centimeter dimensions tend to have greater sensitivity. These ME sensors also have increased sensitivity at their mechanical resonant modes, where the transfer of magnetostrictive strain to the piezoelectric laminate is enhanced. The longitudinal, resonant frequency of such symmetric ME sensors is of order 10 or more $\mathrm{kHz}$. The bending, resonant mode of such unloaded asymmetric sensors in a cantilever configuration is often in the range of hundreds of $\mathrm{Hz}$. In common with other sensors, ME sensors experience $1 / f$ noise that hampers their ability to detect fields at frequencies below several Hz. It is desirable to shift the operating frequency to a higher value where this noise is lower. The signal to noise ratio would be further improved if this shifted, higher operating frequency is equal to the resonant frequency where the ME sensors are more sensitive.

It has been demonstrated that one can increase the operating frequency of magnetic sensors through modulation. ${ }^{2,3}$ In small sensors, such as magnetoresistive sensors, this is done by using movable flux concentrators on microelectromechanical systems (MEMS) structures to modulate the magnetic field

\footnotetext{
${ }^{\text {a) }}$ Author to whom correspondence should be addressed. Electronic mail: alan.s.edelstein.civ@mail.mil.
}

at the position of the sensor. The field modulation increases the operating frequency of the sensor to tens of $\mathrm{kHz}$. It is very difficult to use MEMS flux concentrators to modulate the magnetic field of centimeter sized sensors; the greater mass of large flux concentrators hinders high frequency, oscillatory motion. Modulation frequencies of $100 \mathrm{~Hz}$ were achieved using flux concentrators on a rotating disc, ${ }^{4}$ but there was too large an increase in the background noise generated by acoustic vibrations. An alternative approach is to thermally modulate flux concentrators, ${ }^{5}$ which is difficult at high frequencies for large flux concentrators. Others have constructed a magnetic sensor which combines piezoelectric and magnetostrictive materials. ${ }^{6}$ In this case, the modulation was driven by applying an AC electric field to the piezoelectric material.

Here, we present a method, based on applying a time varying magnetic field, for increasing the operating frequency of ME sensors that incorporates the nonlinear response of magnetostrictive materials. ${ }^{7}$ In its simplest form, for itinerate systems, ${ }^{8,9}$ this response is given by

$$
\frac{\Delta V}{V}=\frac{V \kappa}{2 D \mu_{B}^{2}} \frac{\partial \ln D}{\partial \ln V} M^{2},
$$

where $\mathrm{V}, \kappa, \mathrm{D}, \mathrm{M}$, and $\mu_{\mathrm{B}}$ are volume, the isothermal compressibility, the electronic density of states at the Fermi energy, magnetization density, and Bohr magneton, respectively. We shall consider the case where $M$ is proportional to the field $H$ and take

$$
H(t)=H_{s} \operatorname{Sin} \omega_{s} t+H_{m} \operatorname{Sin} \omega_{m} t, \quad H_{s} \ll H_{m},
$$

where $H_{s}, H_{m}, \omega_{s}$, and $\omega_{m}$ are the magnetic signal amplitude to be measured, the magnetic modulation amplitude, signal frequency, and modulation frequency, respectively. Substituting Eq. (2) into Eq. (1), one finds that there will be a cross term in the magnetostrictive response of the form 
$H_{s} H_{m} \operatorname{Sin} \omega_{s} t \operatorname{Sin} \omega_{m} t$. The transfer of this strain to the piezoelectric layer(s) creates sidebands in the ME output at $\omega_{m} \pm \omega_{s}$ with amplitudes proportional to $H_{s} H_{m}$.

\section{EXPERIMENTAL}

We used a coil surrounding a ME sensor to generate an AC modulation field directed along the measurement direction of the sensor. We investigated both a symmetric and asymmetric ME sensor. The symmetric sensor consisted of a layer of longitudinally poled PZT sandwiched between layers of Metglas (Vitrovac 7600 F, Vitrovac Inc. Hanau, German) of length $8 \mathrm{~cm}$ and width $1 \mathrm{~cm}$. The PZT core is a planar bundle of 5 PZT fibers, each with dimensions of $40 \times 2 \times 0.2 \mathrm{~mm}$. The asymmetric sensor, which had dimension $6 \mathrm{~cm} \times 1 \mathrm{~cm} \times 0.5 \mathrm{~cm}$, was self-biased and consisted of three layers of $25-\mu \mathrm{m}$ Metglas/200- $\mu \mathrm{m} \mathrm{Ni} / 300-\mu \mathrm{m} \mathrm{PZT}$, all mechanically coupled with epoxy. Electrical leads were silverepoxied to the PZT, which was poled perpendicular to the length of the laminate. It was fixed at one end in a cantilever configuration. The Ni provided a field to bias the ME sensor. ${ }^{10,11}$

The measuring system consisted of a set of Helmholtz coils, powered by an Agilent $33220 \mathrm{~A}$ signal generator that was used to apply a sinusoidal, time varying field $H_{s}$ along the longitudinal axis of the sensor. In these experiments, we did not employ any flux concentrators. The signal was detected first using a charge coupled amplifier, PCB Piezotronics Model 441A101, and then filtered in a SR640 lowpass filter with no gain before being sent either to a spectrum analyzer that uses a LabVIEW VI program or to a SR830 DSP Lock-In Amplifier. Resonance frequencies for each cantilever setup were obtained by sweeping the frequency of a 0.0162 Oe external field at progressively finer intervals around the peak value. All the measurements were conducted in our laboratory without the benefit of magnetic, electric, and thermal shielding. Acoustic isolation was provided by a Hertz DT-M vibration isolator.

\section{RESULTS AND DISCUSSION}

The ME response after applying a signal field $H_{s}$ of $0.0162 \mathrm{Oe}$ at $140 \mathrm{~Hz}$ as a function of DC bias field is shown in Fig. 1 for both the asymmetric and symmetric sensors. Both responses are strongly dependent on the DC field and are approximately symmetric functions of this bias. The asymmetric sensor was designed to have a local maximum sensitivity at zero DC field due to the residual magnetism of the $\mathrm{Ni}$ adjacent to the Metglas. The magnetic properties of the Ni layer make the ME response to an additional applied field complicated. To modulate each sensor optimally, the magnitude of the AC modulation field $H_{m}$ was chosen so that the ME output of the sensor at a fixed $H_{s}$ oscillated between its minimum value and a much larger value.

Figure 2 shows the output power spectrum of the symmetric sensor in which a relatively large AC modulation field $H_{m}$ was applied at two different frequencies. In both cases, $H_{m}$ was $0.72 \mathrm{Oe}$ and the signal field $H_{s}$ was $0.0162 \mathrm{Oe}$ at $1.019 \mathrm{~Hz}$. In the first case, $H_{m}$ was applied at $140 \mathrm{~Hz}$. One sees three main peaks corresponding to the modulation frequency $f_{m}$ and the two sidebands $f_{m} \pm f_{s}$. In the second case,
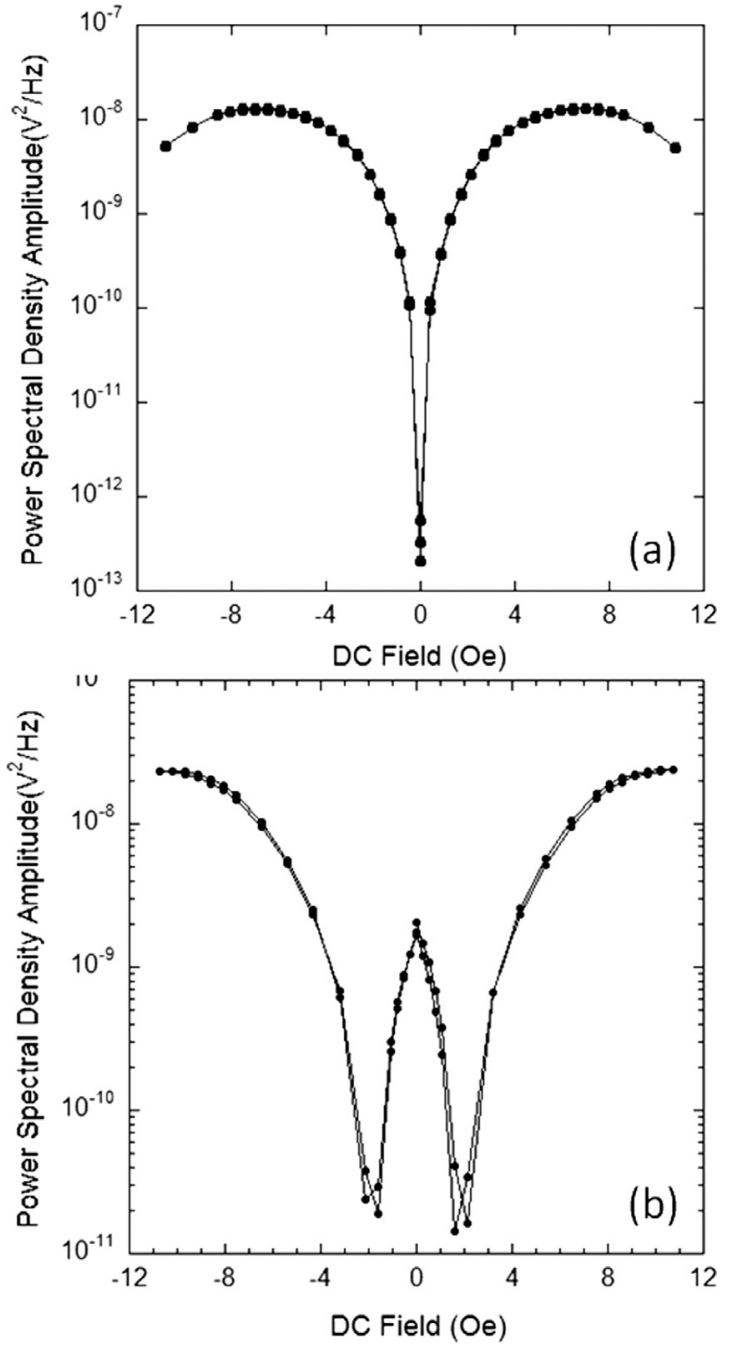

FIG. 1. ME response versus DC biasing fields for the (a) symmetric and (b) asymmetric sensor in a 0.0162 Oe field at $140 \mathrm{~Hz}$.

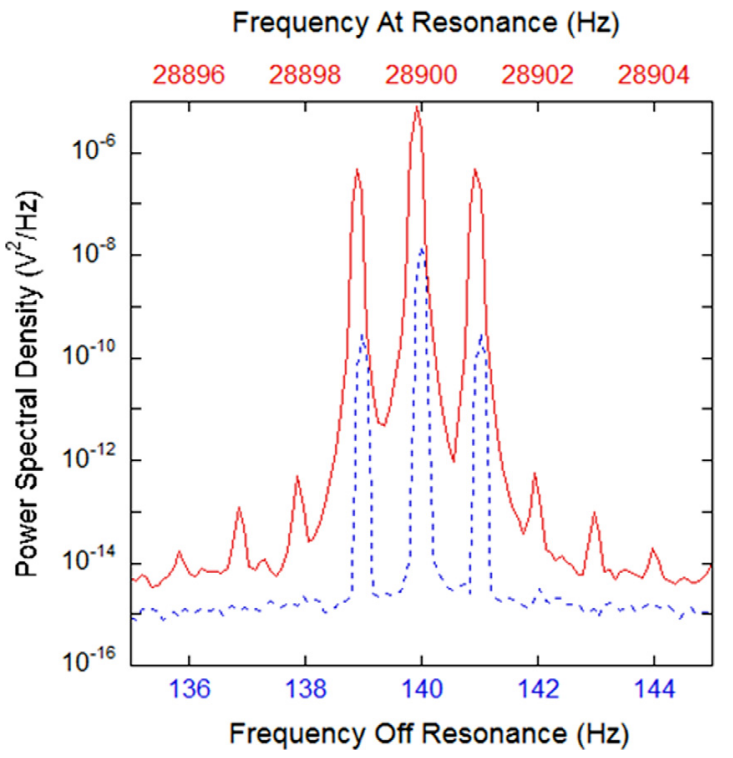

FIG. 2. (Color online) Power spectra from the symmetric sensor detecting a $1.019 \mathrm{~Hz}, 0.0162 \mathrm{Oe}$ field while modulating the sensor with a $0.72 \mathrm{Oe}$ field either off resonance at $140 \mathrm{~Hz}$ (dashed curve) or on resonance at $28.9 \mathrm{k} \mathrm{Hz}$ (solid curve, top x-scale). 


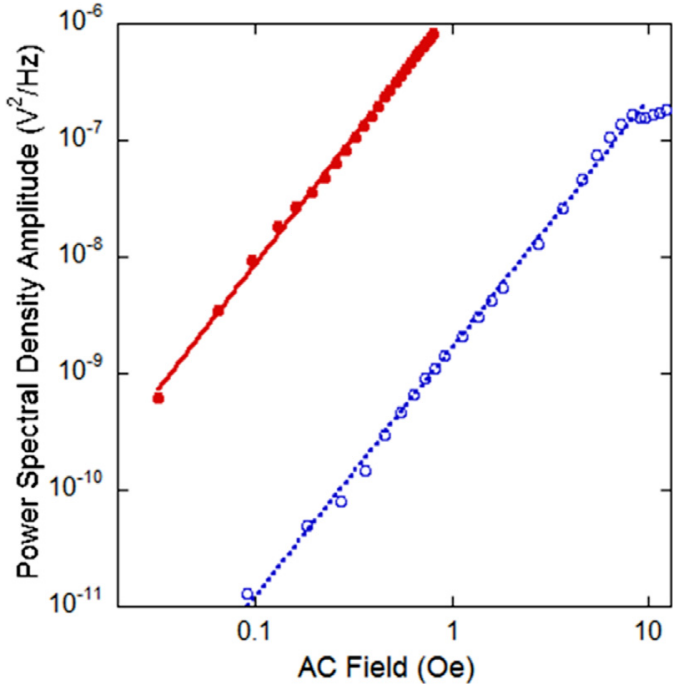

FIG. 3. (Color online) Sideband amplitude in a 0.0162 Oe field at $1.019 \mathrm{~Hz}$ as a function of the AC modulation field $H_{m}$ in the cases of being off resonance at $140 \mathrm{~Hz}$ (dashed curve) or on resonance at $28.9 \mathrm{kHz}$ (solid curve).

the modulation frequency was chosen so that the $f_{m}-f_{s}$ sideband was equal to the longitudinal resonant frequency of the sensor, $28.9 \mathrm{kHz}$. In this case, all the peaks, including the additional sidebands due to the anharmonicity of the sensor, were enhanced by the increased sensitivity of the sensor around the resonance frequency. The amplitude of the side band in the power spectrum versus $H_{m}$ is shown Fig. 3. Both the cases of being on and off resonance are shown. One sees that in either case, the output, as discussed above, is proportional to $H_{m}$ and the power spectrum is proportional to $\mathrm{H}_{m}{ }^{2}$. Note that in the off resonance case, the output deviates from being proportional to $\mathrm{H}_{m}{ }^{2}$ at high values. Since the background around the modulation frequency increases rapidly at higher values of $H_{m}$, there is an optimum value of $H_{m}$ for maximizing the signal to noise ratio.

Figure 4 shows a comparison between the sensor's direct output power spectrum using a DC bias field of 7.0 Oe and the

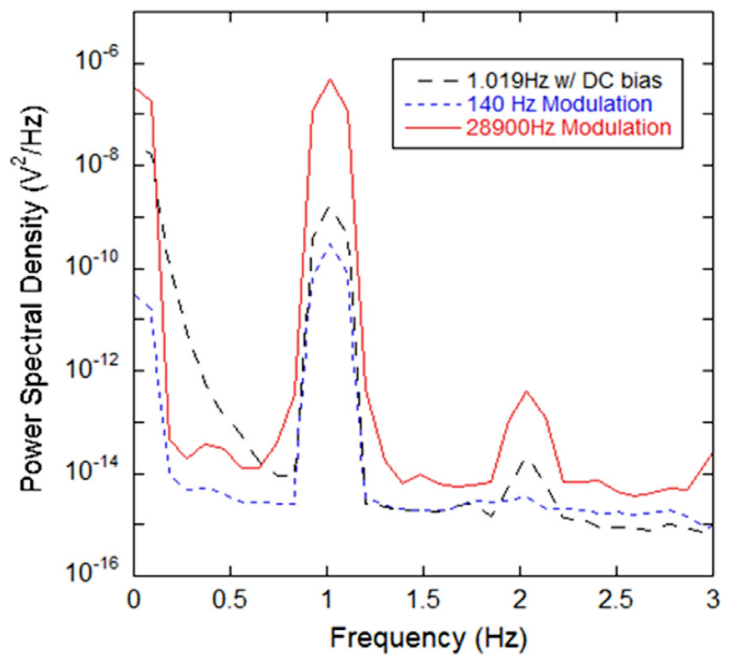

FIG. 4. (Color online) Power spectra from the symmetric sensor detecting a $1.019 \mathrm{~Hz}, 0.0162 \mathrm{Oe}$ field with the direct signal (curve with long dashes), the demodulated signal after modulating off resonance at $140 \mathrm{~Hz}$ (curve with short dashes), and the demodulated signal after modulating on resonance at $28.9 \mathrm{kHz}$ (solid curve).

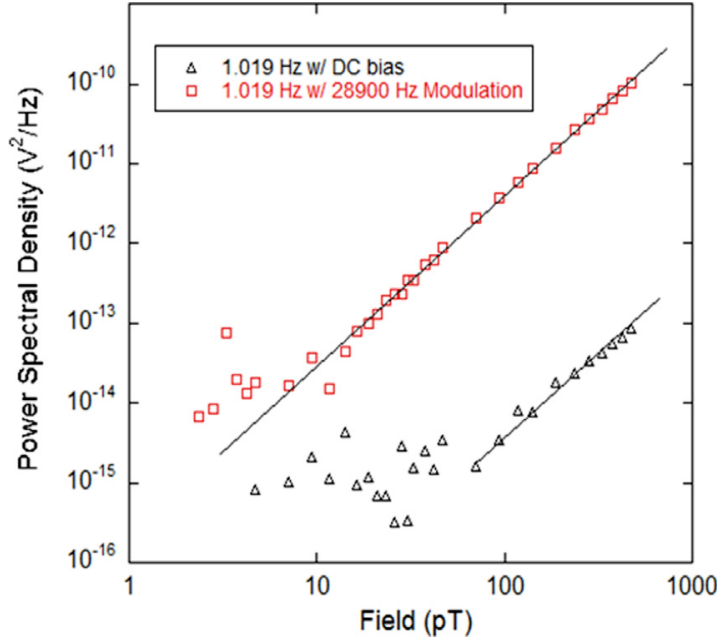

FIG. 5. (Color online) Comparison of the direct signal (squares) and the demodulated sideband signal (triangles) from the symmetric ME sensor at resonance as a function of signal field $H_{s}$.

demodulated signals from $H_{m}$ at the two frequencies discussed above. One sees there is approximately a two orders of magnitude increase in the signal to noise ratio when the sideband frequency is equal to the resonant frequency of the sensor. In Fig. 5, the direct output from a $1.019 \mathrm{~Hz}$ signal field with a DC bias of $7.0 \mathrm{Oe}$ and the demodulated output from a $0.72 \mathrm{Oe}$ modulation field at $28.9 \mathrm{k} \mathrm{Hz}$ are plotted as a function of $H_{s}$. In the modulated case, no DC bias was employed. One sees an increase in field detectivity of the symmetric sensor of a factor of 10 obtained by the modulation technique. We were able to detect a $7 \mathrm{pT} / \sqrt{\mathrm{Hz}}$ field at $1 \mathrm{~Hz}$ with modulation as opposed to a $90 \mathrm{pT} / \sqrt{\mathrm{Hz}}$ field at $1 \mathrm{~Hz}$ without modulation in a magnetically unshielded environment.

Figure 6 shows the output power spectrum of the asymmetric sensor without field modulation resulting from applying an $H_{s}$ of $0.0162 \mathrm{Oe}$ at $1.019 \mathrm{~Hz}$ with an additional optimized 8.2 Oe DC bias field. Figure 7 shows the power spectrum of the asymmetric sensor in the same $1.019 \mathrm{~Hz} H_{s}$

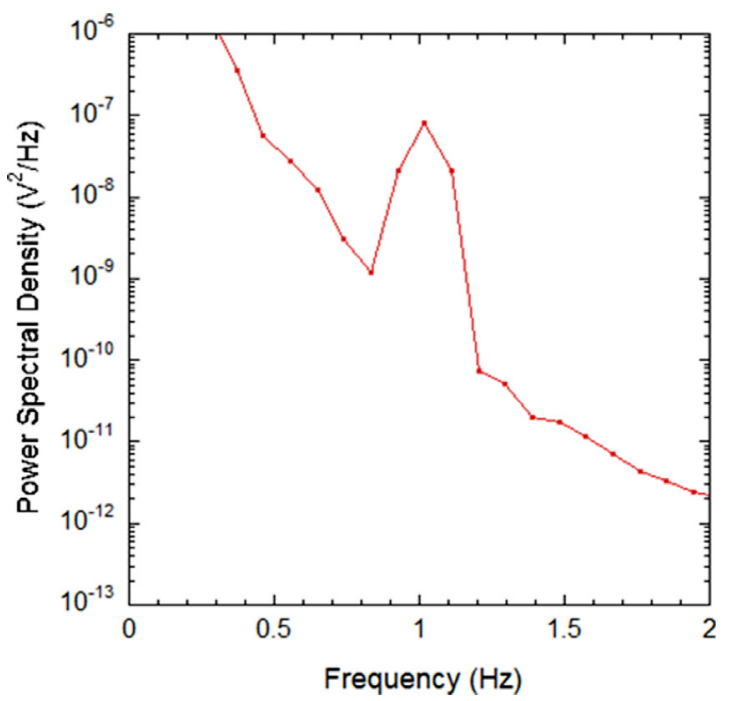

FIG. 6. (Color online) Power spectrum showing the direct detection of a 0.0162 Oe field at $1.019 \mathrm{~Hz}$ using the asymmetric ME sensor with an optimized DC bias of $8.2 \mathrm{Oe}$. 


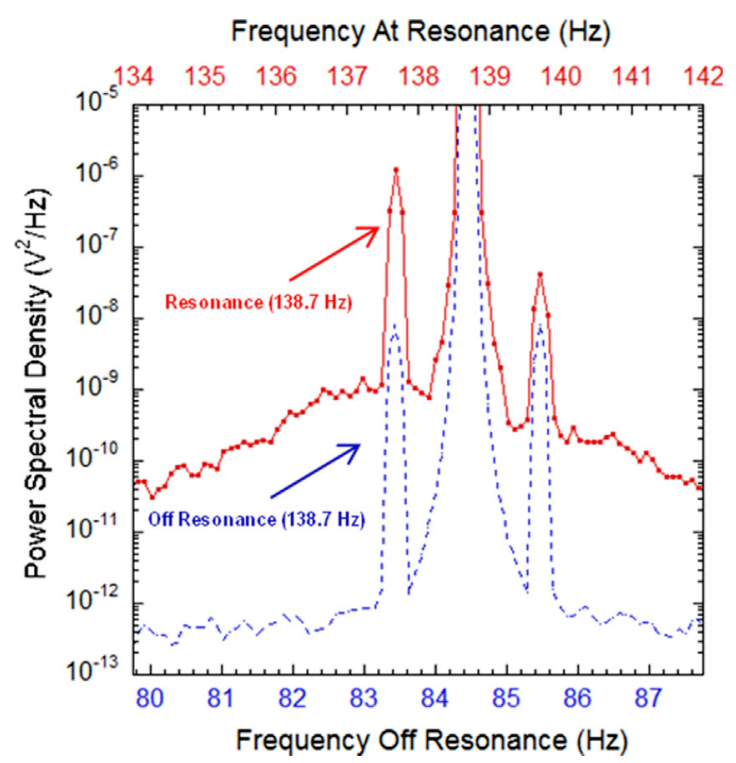

FIG. 7. (Color online) Power spectra from the asymmetric sensor detecting a $1.019, \mathrm{~Hz} 0.0162 \mathrm{Oe}$ field while modulating the sensor off resonance with a modulation frequency of $89.44 \mathrm{~Hz}$ (dashed curve, bottom x scale) and with the lower frequency sideband adjusted to be at the mechanical bending mode frequency of $138.70 \mathrm{~Hz}$ (solid curve, top x-scale).

field but with two different frequency modulation fields. In both cases, there was a DC bias field of 4.6 Oe and an AC modulation field of $3.6 \mathrm{Oe}$. The frequency of this modulation field was either set at $84.4 \mathrm{~Hz}$, which is far below the $138.7 \mathrm{~Hz}$ bending mode resonance of the sensor, or at $139.7 \mathrm{~Hz}$, which is $1.019 \mathrm{~Hz}$ above the bending mode, resonant frequency. Thus, in the later case, the lower frequency sideband $f_{m}-f_{s}$ was at the resonant frequency. It should be pointed out that the modulation field acts on both the magnetostrictive material and the Ni biasing ferromagnet. In the off resonance case at $84.4 \mathrm{~Hz}$, the reduced $1 / f$ noise was responsible for the increase in signal to noise ratio by 2 orders of magnitude relative to the unmodulated signal. On resonance, the lower frequency sideband was increased relative to the higher frequency sideband by the increased sensitivity at the resonance frequency. In contrast to the equal amplitude of the sidebands shown previously for the symmetric sensor, the amplitudes of the sidebands are unequal. The sidebands at resonance for the symmetric sensor are equal because both are within the width of the resonance. Because the resonant frequency of the asymmetric sensor is much lower than the resonant frequency of the symmetric sensor and the two quality factors are comparable, the higher frequency sideband of the asymmetric sensor is not within the resonant peak and, thus, is smaller. The improvement of the lower frequency sideband amplitude for the asymmetric sensor relative to the direct signal is about an order of magnitude. If the background were not increased by the modulation, the signal to noise ratio in the power spectrum of the lower frequency sideband would be 4 orders of magnitude larger than the direct signal and the voltage gain 100 times the direct signal. Thus, it is important to understand and, if possible, decrease the background when the field is modulated near resonance.

\section{CONCLUSION}

In summary, we have demonstrated that applying an oscillating magnetic field to ME sensors increases their operating frequency and their sensitivity. The signal to noise ratio of the sidebands at these higher frequencies is increased because of the lower $1 / f$ noise and, in some cases, because one can take advantage of the larger response at the mechanical resonant frequency. In the best case, for the symmetric sensor on resonance, the signal to noise ratio in the power spectrum was increased by two orders of magnitude. Because of this increase in detectivity, we were able to sense $7 \mathrm{pT} / \sqrt{\mathrm{Hz}}$ at $1 \mathrm{~Hz}$ in a magnetically unshielded environment. A related work, which we have recently become aware of, did not report such high sensitivity at $1 \mathrm{~Hz}^{12}$ In addition, it should be noted that one can also apply a modulation field to very soft flux concentrators and biasing magnets, although the hysteretic effects in bias magnets may increase the background noise significantly. If one can decrease the background noise near resonance when the AC field is applied, then the sensitivity can be further enhanced.

\section{ACKNOWLEDGMENTS}

The support for this project under the Defense Advanced Research Projects Agency (DARPA) Uncooled Magnetic Sensor, Heterostructural Uncooled Magnetic Sensors (HUMS) program is gratefully acknowledged.

${ }^{1}$ J. Zhai, Z. Xing, S. Dong, J. Li, and D. Vieland, J. Am. Ceram. Soc. 91(2), 351 (2008).

${ }^{2}$ A. S. Edelstein, G. A. Fischer, M. Pedersen, E. R. Nowak, and S. F. Cheng, J. Appl. Phys. 99, 08B317/1 (2006).

${ }^{3}$ W. F. J. Egelhoff, P. W. T. Pong, J. Unguris, R. D. McMichael, E. R. Nowak, A. S. Edelstein, G. A. Fischerd, Sens. Actuators, A 155, 217 (2009).

${ }^{4}$ Unpublished ARL data, 2010

${ }^{5}$ W. Wang and Z. Jiang, IEEE Trans. Magn. 44, 4003 (2008).

${ }^{6}$ M. D. Mermelstein and A. Dandridge, Appl. Phys. Lett. 51(7), 545 (1987).

${ }^{7}$ A. Pantinakis and D. A. Jackson, J. Appl. Phys. 65(7), 2872 (1989).

${ }^{8}$ M. Doerr, M. Rotter, and A. Lindbaum, Adv. Phys. 54(1), 1 (2005).

${ }^{9}$ J. F. Janak and A. R. Williams, Phys. Rev. B 14(9), 4199 (1976).

${ }^{10}$ S. K. Mandal, G. Sreenivasulu, V. M. Petrov, and G. Srinivasan, Appl. Phys. Lett. 96(19), 192502 (2010).

${ }^{11}$ S.-C. Yang, C.-S. Park, K.-H. Cho, and S. Priya, J. Appl. Phys. 108(9), 093706 (2010).

${ }^{12}$ S. M. Gillette, A. L. Geiler, D. Gray, D. Viehland, C. Vittoria, and V. G. Harris, IEEE Magn. Lett. 1, 1 (2011). 